POS $\quad$ PROCEEDINGS

\title{
Tau neutrino appearance with KM3NeT/ORCA
}

\section{Thomas Eberl}

Universität Erlangen-Nürnberg, Erwin-Rommel-Str. 1, 91058 Erlangen, Germany

Erlangen Centre for Astroparticle Physics (ECAP)

E-mail: thomas.eberlefau.de

\section{Steffen Hallmann*}

Universität Erlangen-Nürnberg, Erwin-Rommel-Str. 1, 91058 Erlangen, Germany

Erlangen Centre for Astroparticle Physics (ECAP)

E-mail: steffen.hallmannefau.de

\section{Jannik Hofestädt}

Universität Erlangen-Nürnberg, Erwin-Rommel-Str. 1, 91058 Erlangen, Germany

Erlangen Centre for Astroparticle Physics (ECAP)

E-mail: jannik.hofestaedtefau.de

\section{on behalf of the KM3NeT Collaboration}

KM3NeT/ORCA is a mega-ton volume neutrino detector and has entered the construction phase in the Mediterranean Sea. Optimised to study neutrino oscillations in the few GeV energy range, it has unprecedented sensitivity to oscillations of atmospheric muon and electron neutrinos into the tau neutrino channel. Tau neutrinos predominantly produce shower-like signatures, which can be identified on a statistical basis as an energy- and direction-dependent excess over the nonappearance hypothesis.

The contribution shows that the KM3NeT/ORCA detector will be able to exclude non-appearance already in the first months of data taking. In the longer run precise measurement of the tau normalisation will allow to put constraints on the commonly assumed unitarity of three neutrino mixing.

35th International Cosmic Ray Conference - ICRC2017

10-20 July, 2017

Bexco, Busan, Korea

\footnotetext{
${ }^{*}$ Speaker.
} 


\section{Current status of neutrino mixing}

Today it is well established that the three known neutrino flavour eigenstates $\left(v_{e}, v_{\mu}, v_{\tau}\right)$ oscillate as admixtures of three mass eigenstates $\left(v_{1}, v_{2}, v_{3}\right)$. Under the assumption that this picture is complete, the mixing between flavour and mass eigenstates is unitary, meaning that no neutrinos oscillate into yet unknown flavour eigenstates during propagation. The mixing can be described by three mixing angles $\left(\theta_{12}, \theta_{13}, \theta_{23}\right)$, one charge parity violating phase $\left(\delta_{\mathrm{CP}}\right)$ and the two squared mass splittings $\left(\Delta m^{2}, \Delta M^{2}\right)$ between the mass eigenstates. Thanks to matter effects in the Sun, [1] solar neutrino experiments were able to measure the sign of the small mass splitting between the first and the second mass eigenstate $\Delta m^{2}=m_{2}^{2}-m_{1}^{2}>0[2,3]$, while for the much larger atmospheric mass splitting only the absolute value could be determined $\left|\Delta M^{2}\right|=\left|m_{3}^{2}-m_{1}^{2}\right| \approx$ $\left|m_{3}^{2}-m_{2}^{2}\right|$ [4]. The undetermined sign of the large mass splitting is commonly referred to as the mass ordering problem, which could be either normal for $m_{1}<m_{2}<m_{3}$ or inverted ordering for $m_{3}<m_{1}<m_{2}$. Furthermore, vanishing $\delta_{\mathrm{CP}}$ is currently only disfavoured at the $2 \sigma$ level and its value remains undetermined. Also the octant of $\theta_{23}$, which affects strongly the sensitivity of future experiments to determine the mass hierarchy, is not known [5]. All other neutrino oscillation parameters have been determined with reasonable accuracy and yield a consistent picture. Hence, next generation experiments will need to perform precision measurements to reduce uncertainties of the oscillation parameters and to probe deviations from the commonly accepted picture of unitary $3 \times 3$ neutrino oscillation.

Beam-line and reactor neutrino experiments measure oscillation probabilities by studying the appearance or disappearance of a certain neutrino flavour on the path from the source to the detector. Due to the large rest mass of the $\tau$ lepton, the $v_{\tau}$ charged current (CC) cross section has a threshold of $3.4 \mathrm{GeV}$ and only reaches $40 \%$ of the $v_{\mu} \mathrm{CC}$ cross section at $20 \mathrm{GeV}$ [6]. The main signal of $v_{\tau}$ appears in the range between 10 and $30 \mathrm{GeV}$, where the conversion from $v_{\mu} \rightarrow v_{\tau}$ has a broad maximum for neutrinos that have traversed the Earth. To be able to observe the appearance of $v_{\tau}$, a high energy neutrino beam is therefore required. It is because beam-line experiments often operate at lower energies and the energy threshold of large volume neutrino telescopes is too high that the oscillation into the $v_{\tau}$ sector is currently the least constrained [7].

While the direct appearance of $v_{\tau}$ has already been observed by other experiments (see Sec. 2), the KM3NeT/ORCA detector presented in Sec. 3 will collect unprecedented statistics of $v_{e}$ and $v_{\mu}$ produced in the atmosphere that have oscillated into the $v_{\tau}$ channel. After an explanation of the measurement in Sec.4, we show in Sec. 5 that KM3NeT/ORCA will be able to determine the normalisation of the oscillated $v_{\tau}$ flux with high precision. In the long term, this allows to probe deviations from the unitarity assumption of three neutrino mixing which would be a clear hint that the current picture of the neutrino sector is incomplete and new physics is at play $[8,9]$.

Note that throughout this proceeding the term neutrinos implicitly includes also anti-neutrinos.

\section{Existing and future $v_{\tau}$ appearance measurements}

The appearance of $v_{\tau}$ has been directly detected in the neutrino beam from CERN to Gran Sasso with the OPERA detector. Thanks to the excellent vertex resolution of the detector, OPERA can distinguish $v_{\tau}$ events from other interaction channels by identifying the small kink between 
the primary vertex in the CC interaction $\left(v_{\tau}+\right.$ nucleon $\rightarrow \tau^{-}+$hadronic cascade) and the second vertex produced by the rapid $\tau$-lepton decay. In this way it was possible to obtain a significance of $5.1 \sigma$ with only five $v_{\tau}$-like events [10]. However, the limited detector mass and small interaction cross sections at low energies complicate the measurement of small deviations from the expected $v_{\tau}$ flux normalisation. For the collection of high $v_{\tau}$ statistics much larger and consequently less densely instrumented neutrino detectors that use the atmospheric neutrino flux are preferred. While OPERA was able to identify $v_{\tau}$ events on an event-by-event basis thanks to its millimeter precision vertex resolution, large scale detectors for atmospheric neutrinos can only measure $v_{\tau}$ interactions on a statistical basis by observing an excess in the count rate with respect to the non-appearance assumption.

The underground Super Kamiokande detector has been taking data with its instrumented volume of 50 kilo-tons for more than a decade. Using multivariate techniques to improve the signal significance, they can in the latest update of their analysis observe $v_{\tau}$ appearance with almost $5 \sigma$ [11], retaining a $v_{\tau}$ contribution of only about 20 events per year in the analysis sample [12].

The $\sim 10 \mathrm{GeV}$ energy threshold of DeepCore, the low-energy extension of the IceCube neutrino observatory, is also low enough to probe $v_{\tau}$ appearance and a sensitivity close to $5 \sigma$ for the exclusion of non-appearance can be achieved [13]. Thanks to the better resolution and larger instrumented volume with respect to DeepCore, the proposed dense in-fill array PINGU is able to constrain the normalisation of the $v_{\tau}$ flux to $\pm 40 \%$ (with $5 \sigma$ significance) within one year of operation [14]. In the following, we show that a comparable performance can be achieved by the KM3NeT/ORCA detector. A comparison of the measured flux normalisations and sensitivities is shown in Fig. 1.

Figure 1: $90 \%$ confidence level bands of the current OPERA [10], Super Kamiokande [12, scaled to 90\% CL.] $v_{\tau}$ flux normalisation measurements and the corresponding sensitivities of DeepCore [13], Pingu [14] and KM3NeT/ORCA (this work).

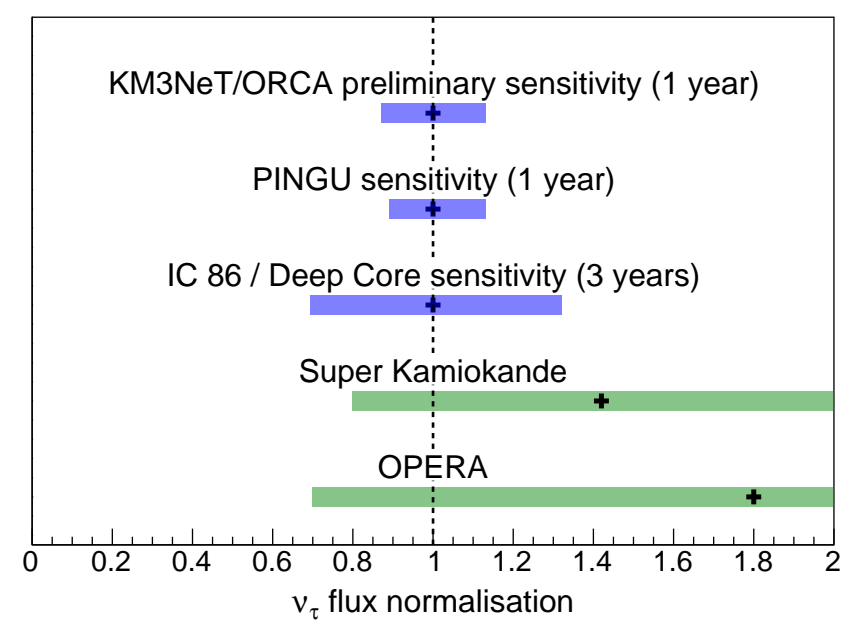

\section{KM3NeT/ORCA - oscillation studies in the deep-sea}

KM3NeT is a distributed research infrastructure under construction in the Mediterranean Sea [15]. The low-energy branch of it, the KM3NeT/ORCA detector, will be deployed in a depth of $2.5 \mathrm{~km}$ offshore Toulon in France. It consists of 115 detection strings which are anchored to the sea bed and held upright by a submarine buoy. Each detection string houses 18 Digital Optical Modules (DOMs) based on 31 small 3 inch photomultiplier tubes to detect the Cherenkov light induced by 
charged secondary particles from neutrino interactions. With a horizontal inter-string spacing of about $20 \mathrm{~m}$ and a vertical spacing of $9 \mathrm{~m}$ between DOMs, more than six mega-tons of sea water will be instrumented with optical sensors for the reference detector layout [15]. ${ }^{1} \mathrm{KM} 3 \mathrm{NeT} / \mathrm{ORCA}$ is optimised to study the oscillation of neutrinos produced in the atmosphere with an energy threshold of few GeV. Its primary goal is the determination of the neutrino mass ordering. Thanks to the large instrumented volume, KM3NeT/ORCA will be able to collect large statistics of oscillated atmospheric neutrinos and thus provide stringent results also on other oscillation parameters. One of the early physics measurements of KM3NeT/ORCA will be the appearance of $v_{\tau}$.

\section{Measurement of $v_{\tau}$ appearance using atmospheric neutrinos}

High energy cosmic rays permanently impinge on the Earth's upper atmosphere where they interact and produce cascades of secondary particles. In these cascades the decays of charged pions and kaons give rise to an atmospheric neutrino flux of $v_{\mu}$ and $v_{e}$. At production, the contribution of $v_{\tau}$ to the atmospheric flux is negligibly small as they can only be produced in the decay of mesons containing heavy quarks such as $c$ and $b$. A measured $v_{\tau}$ component at the detector appears therefore only due to neutrino oscillation. As mentioned above, the $v_{\tau} \mathrm{CC}$ cross section amounts to $\sim 40 \%$ of $v_{e, \mu} \mathrm{CC}$ in the energy region of total $v_{\mu} \rightarrow v_{\tau}$ conversion at $\approx 25 \mathrm{GeV}$. These energies are well above the threshold of detection for KM3NeT/ORCA.

In the $\mathrm{CC}$ interaction of the muon neutrino, an outgoing muon is produced which travels more than $4 \mathrm{~m} / \mathrm{GeV}$ while inducing Cherenkov radiation. These events therefore predominantly leave a track-like event topology in the detector. In contrast, electrons and hadronic cascades produced in $v_{e} \mathrm{CC}$ and neutral current (NC) interactions appear more spherical, i.e. shower-like. The $\tau$ lepton produced in $v_{\tau}$ CC interactions decays instantly and only appears track-like in its decay to a muon (17\% branching fraction). This means that events are transferred from the track to the shower channel thanks to the $v_{\mu} \rightarrow v_{\tau}$ oscillation. Consequently, $v_{\tau}$ appearance can be observed as a statistical excess of shower-like events compared to the non-appearance scenario.

A trained classifier is used in KM3NeT/ORCA to assign the reconstructed events to one of these two categories. As can be seen from Fig. 2, for an example energy of $15 \mathrm{GeV}$ more than $80 \%$ of all $v_{\tau}$ events are classified as showers. In contrast, only $10 \%\left(\bar{v}_{\mu}\right)$ to $30 \%\left(v_{\mu}\right)$ of the muon neutrinos fall in this category.

Every year $2.5 \times 10^{3} v_{\tau}$ CC events will be recorded and classified as shower by KM3NeT/ORCA. The rate of other neutrino interactions classified as shower is an order of magnitude higher $\left(17 \times 10^{3}\right.$ $v_{e} \mathrm{CC}, 11 \times 10^{3} v_{\mu} \mathrm{CC}$ and $6 \times 10^{3} \mathrm{NC}$ events per year) but peaks at lower energies. Because of the dense instrumentation, the contribution of atmospheric muons in the final event sample is small [15].

Looking at the distribution in reconstructed energy and cosine of the zenith angle, the statistical signal significance of the $v_{\tau}$ appearance measurement in each bin $i$ can be expressed by the

\footnotetext{
${ }^{1}$ The actual detector will have slightly larger horizontal spacing and therefore larger instrumented volume. Improvements in trigger and reconstruction result in a lower threshold energy and same resolution despite the sparser instrumentation compared to the reference detector. The results presented here are therefore conservative. [16]
} 


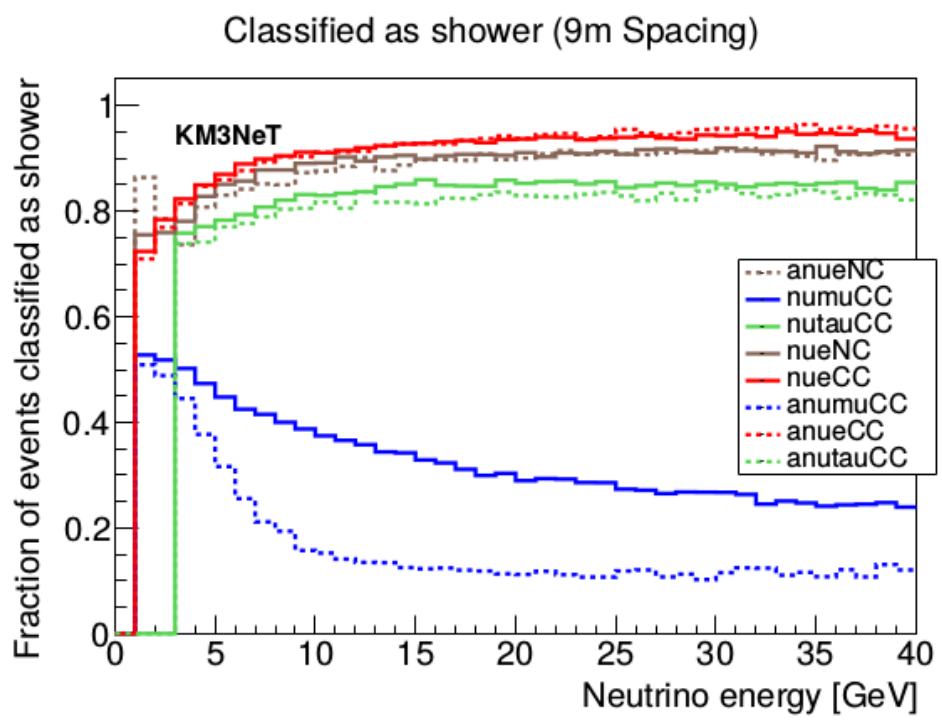

Figure 2: Fraction of events classified as shower in KM3NeT/ORCA as a function of neutrino energy. Figure taken from Ref. [15].

following quantity:

$$
S_{i}=\frac{N_{i}\left(v_{\tau} \mathrm{CC}\right)}{\sqrt{N_{i}(\text { total })}},
$$

which represents a statistical per-bin signal significance in terms of sigmas. This quantity is shown in Fig. 3. The region of maximum significance traces the oscillation maximum of $v_{\mu} \rightarrow v_{\tau}$, i.e. the region around $25 \mathrm{GeV}$ for straight up-going neutrinos is most important for the $v_{\tau}$ appearance measurement. In the reconstructed energy the most significant region is slightly shifted to lower energies with respect to the oscillation maximum which is in part due to the un-seen energy carried away by the additional neutrinos in $\tau$-decay. In the presented significance, the reconstructed event distribution is obtained by two-dimensional and flavour-dependent smearing in angle and energy of the true distributions. The same procedure was followed in the sensitivity studies for the mass ordering determination in Ref. [15].

\section{Sensitivity of ORCA to $v_{\tau}$ appearance}

In order to determine the sensitivity of the KM3NeT/ORCA detector for a measurement of the $v_{\tau}$ flux normalisation a parametrised detector response has been used. A combined $\Delta \chi^{2}$ fit of nuisance and oscillation parameters is then performed on these parametrised energy and zenith angle dependent event distributions. In the fit, the oscillation parameters are either fixed or fitted with or without priors depending on the precision with which they are currently known. More information on the used parameters is given in Tab. 1. Also, several systematic uncertainties are taken into account as nuisance parameters. Besides the relative flux normalisations of the different $\mathrm{CC}$ interaction channels and the NC contribution, these include the uncertainties on the energy slope and the neutrino-antineutrino ratio of the atmospheric flux. The fit result is robust against the octant of $\theta_{23}$ and on the mass ordering. If the ordering used in the fit is opposite of the one realised in nature, the minimum of the $\Delta \chi^{2}$ fit shifts by less than $5 \%$ from the flux normalisation under the unitarity assumption. The fit is repeated for several different operation durations of the experiment. 


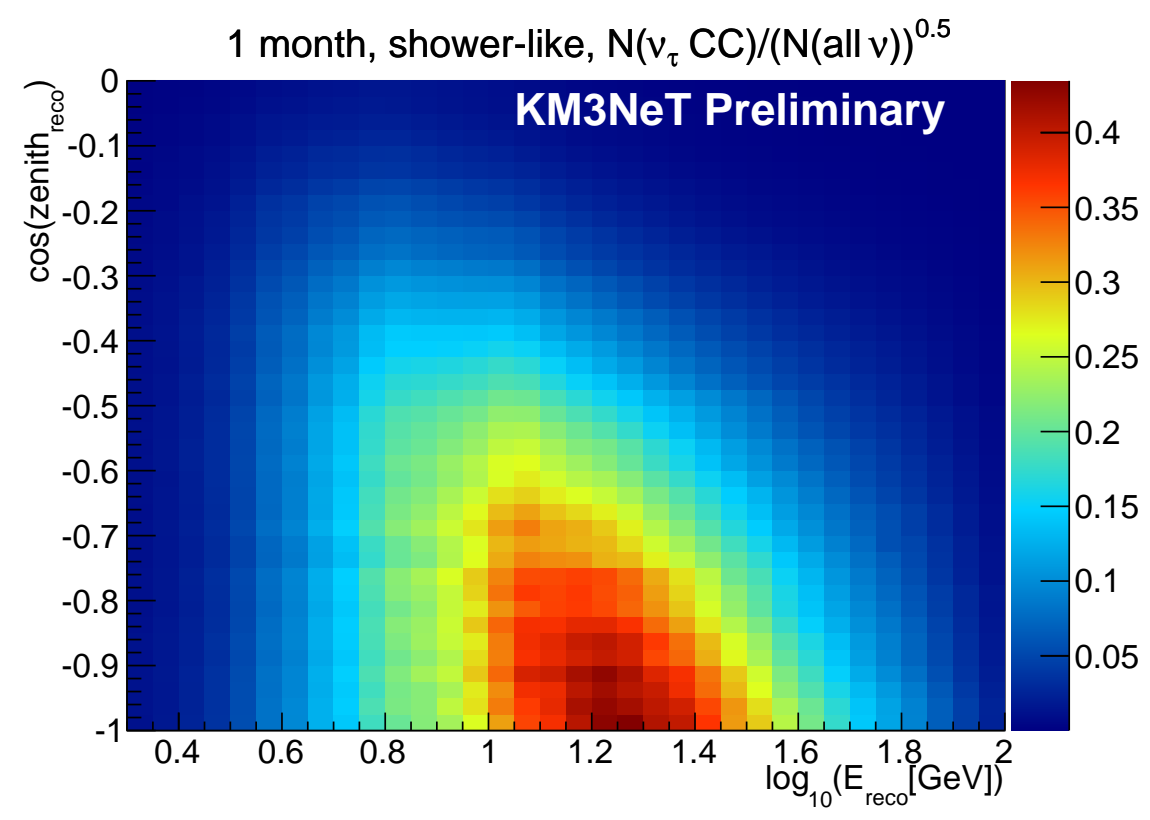

Figure 3: Statistical per-bin significance for the detected $v_{\tau}$-signal in the shower-channel per one month of data taking.

Table 1: Values for the oscillation parameters and nuisance parameters used in the fit. oscillation parameters nuisance parameters

\begin{tabular}{|c|c|c|c|c|c|c|c|}
\hline parameter & true value & prior & start value & parameter & true val. & prior & start val. \\
\hline$\theta_{12}$ & $33.4^{\circ}$ & fixed & fixed & norm $v_{e} \mathrm{CC}$ & 1 & - & 1 \\
\hline$\Delta m^{2}\left[\mathrm{eV}^{2}\right]$ & $7.53 \times 10^{-5}$ & fixed & fixed & norm $v_{\mu} \mathrm{CC}$ & 1 & - & 1 \\
\hline$\theta_{13}$ & $8.42^{\circ}$ & $0.26^{\circ}$ & $8.42^{\circ}$ & norm $v_{\tau} \mathrm{CC}$ & 1 & - & 1 \\
\hline$\theta_{23}$ & $42^{\circ}$ & - & $42^{\circ} / 48^{\circ}$ & norm $v \mathrm{NC}$ & 1 & 0.31 & 1 \\
\hline$\Delta M^{2}\left[\mathrm{eV}^{2}\right]$ & $2.44 \times 10^{-3}$ & - & $2.44 \times 10^{-3}$ & energy slope & 0 & - & 0 \\
\hline$\delta_{\mathrm{CP}}$ & 0 & - & 0 & $v / \bar{v}$ ratio & 0 & 0.1 & 0 \\
\hline
\end{tabular}

The resulting sensitivity of the KM3NeT/ORCA detector to measure the $v_{\tau}$ flux normalisation is shown in Fig. 4. A flux normalisation of 1 is expected for unitary mixing. In case additional today unidentified flavours of neutrinos are present, part of the flux might oscillate into these channels. Consequently, the measured normalisation of the $v_{\tau}$ flux will be smaller than the expectation, i.e. $<1$. In contrast, values much larger than one are unphysical. If such a value is measured, it is likely that the currently assumed $v_{\tau}$ CC cross section is underestimated.

Assuming unitarity holds for the event distribution to be fitted, then already after two months of operation the non-appearance of $v_{\tau}$ can be excluded with a significance of more than $5 \sigma$ (see Fig. 4). In the longer term, KM3NeT/ORCA will then be able to precisely determine the normalisation of the flux. After one year, the normalisation will be constrained to better than $\pm 20 \%( \pm 40 \%)$ with a significance of $3 \sigma(5 \sigma)$ in case of the assumed unitary $3 \times 3$ neutrino mixing.

The authors gratefully acknowledge support by the Deutsche Forschungsgemeinschaft (DFG). 


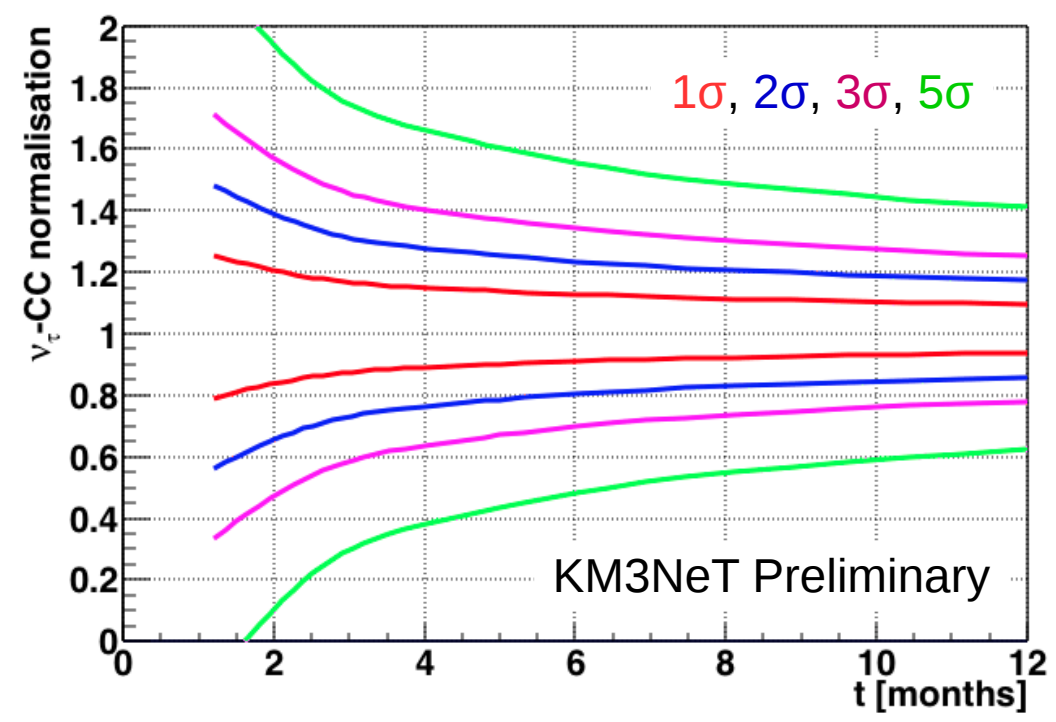

Figure 4: Sensitivity of the KM3NeT/ORCA detector to appearance of $v_{\tau}$ as a function of operation time. A $v_{\tau}$ CC normalisation of one is expected in the case of unitary mixing.

\section{References}

[1] A. Y. Smirnov, The MSW effect and solar neutrinos, hep-ph/0305106.

[2] Q. R. Ahmad et al., Measurement of the rate of $v_{e}+d \rightarrow p+p+e^{-}$interactions produced by ${ }^{8}$ B solar neutrinos at the Sudbury Neutrino Observatory, Phys. Rev. Lett. 87 (2001) 071301, [nucl-ex/0106015].

[3] Q. R. Ahmad et al., Direct evidence for neutrino flavor transformation from neutral current interactions in the Sudbury Neutrino Observatory, Phys. Rev. Lett. 89 (2002) 011301, [nucl-ex/0204008].

[4] Y. Fukuda et al., Evidence for oscillation of atmospheric neutrinos, Phys. Rev. Lett. 81 (1998) 1562-1567, [hep-ex/9807003].

[5] F. Capozzi, E. Lisi, A. Marrone, D. Montanino, and A. Palazzo, Neutrino masses and mixings: Status of known and unknown $3 v$ parameters, Nucl. Phys. B908 (2016) 218-234, [arXiv:1601.0777].

[6] J. A. Formaggio and G. P. Zeller, From eV to EeV: Neutrino cross sections across energy scales, Rev. Mod. Phys. 84 (2012) 1307-1341.

[7] P. Migliozzi and F. Terranova, Learning from tau appearance, New J. Phys. 13 (2011) 083016, [arXiv:1107.3018].

[8] X. Qian, C. Zhang, M. Diwan, and P. Vogel, Unitarity Tests of the Neutrino Mixing Matrix, arXiv: 1308.5700 . 
[9] S. Verma and S. Bhardwaj, Prospects for Reconstruction of Leptonic Unitarity Quadrangle and Neutrino Oscillation Experiments, Nucl. Phys. B907 (2016) 249-257, [arXiv:1603.0829].

[10] OPERA Collaboration, N. Agafonova et al., Discovery of $\tau$ Neutrino Appearance in the CNGS Neutrino Beam with the OPERA Experiment, Phys. Rev. Lett. 115 (2015), no. 12 121802, [arXiv:1507.0141].

[11] S. Moriyama, New atmospheric and solar results from Super-Kamiokande, Proceedings of the Neutrino2016 (London), to be published.

[12] Super-Kamiokande Collaboration, K. Abe et al., Evidence for the Appearance of Atmospheric Tau Neutrinos in Super-Kamiokande, Phys. Rev. Lett. 110 (2013), no. 18 181802, [arXiv:1206.0328].

[13] M. Larson, Atmospheric tau neutrino appearance measurement with IceCube DeepCore, in Proceedings of the Neutrino2016 conference (London), to be published.

[14] The IceCube-Gen2 Collaboration, PINGU: A Vision for Neutrino and Particle Physics at the South Pole, arXiv:1607.0267.

[15] KM3NeT Collaboration, S. Adrian-Martinez et al., Letter of intent for KM3NeT 2.0, J. Phys. G43 (2016), no. 8 084001, [arXiv: 1601.0745 ].

[16] A. Kouchner for the KM3NeT Collaboration PoS(ICRC2017)1027 (2017). 